\title{
Preliminary assessment of photonic solutions based on C-band VCSELs for multi-Tb/s metro networks
}

\author{
P. Parolari ${ }^{1}$ Senior Member, IEEE, A. Gatto ${ }^{1}$ Member, IEEE, M. Rapisarda ${ }^{1}$, F. Lipparini ${ }^{1}$, C. Neumeyr ${ }^{2}$, \\ M. Svaluto Moreolo ${ }^{3}$ Senior Member, IEEE, P. Boffi ${ }^{1}$ Member, IEEE \\ ${ }^{1}$ Politecnico di Milano, Dip. Elettronica Informazione e Bioingegneria, Via Ponzio 34/5,20133 Milano, Italy \\ ${ }^{2}$ VERTILAS GmbH, Daimlerstr 11d, D-85748 Garching, Germany \\ ${ }^{3}$ Centre Tecnologic de Telecomunicacions de Catalunya (CTTC/CERCA), Av.C. F. Gauss 7, 08860 Castelldefels \\ (Barcelona), Spain \\ e-mail: paola.parolari@polimi.it
}

\begin{abstract}
C-band InP vertical cavity surface emitting laser (VCSEL) exploitation can be appealing also for high-capacity transmission over hundreds of kilometres. Long-wavelength VCSELs can represent an alternative solution for the development of transmitters with reduced cost, power consumption and footprint by adopting direct modulation (DM) and single sideband (SSB) discrete multitone (DMT) modulation to achieve dense wavelength division multiplexing (WDM) granularity. Due to numerous hops between nodes inside metropolitan area networks the effect of filtering can severely impact the transmission performance. We present preliminary experimental assessments of DM VCSEL sources with multi-carrier modulation formats, for more than $50 \mathrm{~Gb} / \mathrm{s}$ per-channel transmission to target a metro network including nodes, handling $25-\mathrm{GHz}$ granularity.
\end{abstract}

Keywords: VCSELs, metro networks, WSS, discrete multitone transmission.

\section{INTRODUCTION}

Nowadays, most of the total data traffic is concentrated in less of the $5 \%$ of the geographical area, for example in the metropolitan area networks (MANs). This kind of networks need to support services such as 5G, Mobile Edge Computing, UHD TV, etc.. generating a huge IP traffic with uneven distribution along day-time. Optical transport technologies for MANs are encouraged to shift the paradigm of long-haul transmission developing agile solutions supporting a "pay-as-you-grow" evolution, to target reduced costs and possibly power consumption [1].

Innovative photonic technologies have the potentials to support the optical data transport allowing a huge transmission capacity and routing over variable distances and topologies, providing also low cost, low power consumption and reduced footprint. Different paths have been chosen to guarantee this high throughput either increasing the number of wavelength-division multiplexed (WDM) channels [2] exploiting ultra-wide band amplification ( $\mathrm{S}, \mathrm{C}$ and $\mathrm{L}$ band) or increasing the single channel data rate exploiting ultra-high speed siliconphotonics ( $\mathrm{SiPh}$ ) devices [3].

As an alternative multi- $\mathrm{Tb} / \mathrm{s}$ capacity can be achieved employing massive photonic integration to develop a photonic integrated circuit (PIC) transmitter including multiple long-wavelength high-bandwidth vertical cavity surface emitting laser (VCSEL) sources and SOI multiplexers [4, 5]. VCSELS have been used for a long time in data communications [6] but, the exploitation of direct modulation (DM) with discrete multitone (DMT) modulation with single-mode devices $[7,8]$ combined with multi-channel coherent receivers (CO-RXs) allows to realize a compact and cost-effective sliceable bandwidth and bitrate variable transceiver (S-BVT) able to adaptively load/manipulate the spectrum, enabling multiple $\mathrm{Tb} / \mathrm{s}$ capacity [9].

Long-wavelength InP VCSELs emitting in the C band, DM by multicarrier formats, such as DMT, have been in fact recently proposed with direct detection (DD) to bridge tens of $\mathrm{km}$ distances with more than $50 \mathrm{~Gb} / \mathrm{s}$ capacities [10-12]. To target typical MAN reach distances in standard single mode fibre (SSMF) links, there is otherwise the necessity to avoid the impairments due to the cumulated chromatic dispersion (CD), which indeed can be compensated by exploiting digital signal processing (DSP) techniques enabled by coherent detection (COHD) [13]. Moreover, in order achieve a higher spectral efficiency and target very dense WDM transmission, i.e. with 25-GHz channel spacing, single side-band (SSB) DMT modulation can be performed [14]. This helps also in improving the resilience towards filtering effects due to traversing multiple MAN nodes, based on wavelength selective switching (WSS) filters, which should be carefully considered due to the adopted fine WDM granularity [15].

In this paper we assess the performance of the above-mentioned transmitter, employing coherent detection with reduced complexity [16]. In particular we compare simulations and experimental results; we evaluate the transported capacity for single channel transmission in function of nodes number, considering the actual VCSEL chirp coefficients and frequency response and we confirm already obtained simulated results [15] demonstrating reach distances of hundreds of kms typical of MANs, maintaining a capacity per channel higher than $50 \mathrm{~Gb} / \mathrm{s}$. 


\section{C-BAND VCSEL-BASED S-BVT TRANSMITTER}

The motivations which lead to use of a very tight filtering to obtain a DMT SSB modulated signal come from the aim to develop a multi-Tb/s S-BVT transmitter. Thanks to bit and power loading at DSP level, DMT is able to address the efficient usage of the bandwidth resource as a function of the requested capacity allowing dynamic and flexible adaptation to traffic/channel conditions [17]. SSB modulation allows on the other hand to interleave up to 4 SOI chip modules integrating multiple VCSELs in the C-band with 100-GHz channel spacing achieving an ultimate granularity of $25 \mathrm{GHz}$ [1]. By targeting beyond $50-\mathrm{Gb} / \mathrm{s}$ per VCSEL rate the overall supermodule capacity can reach $8-\mathrm{Tb} / \mathrm{s}$ capacity with $4-\mathrm{b} / \mathrm{s} / \mathrm{Hz}$ spectral efficiency in case of polarization division multiplexing (PDM) $[16,18]$.

\subsection{Experimental set up}

In order to have a preliminary assessment of the above-described multi-Tb/s transmitter we evaluated the performance of the single DM VCSEL in presence of propagation in up to $200 \mathrm{~km} \mathrm{SSMF}$ with the use of a Finisar Waveshaper 4000S to mimic the presence of WSSs in the MAN, as depicted in Figure 1.

The short cavity (SC) VCSEL emits at $1533.5 \mathrm{~nm}$, with 5-MHz measured linewidth. It is characterized by an $\mathrm{E} / \mathrm{O}$ bandwidth of $18 \mathrm{GHz}$ and preliminary measurements provided a $\alpha$ linewidth enhancement factor of 3.7 and $\kappa$ adiabatic constant of $1.52 \cdot 10^{13}[15]$.

The DMT signal is composed by 256 sub-carriers in a $20-\mathrm{GHz}$ frequency range, thus the sub-carrier spacing is $78.125 \mathrm{MHz}$; a cyclic prefix (CP) of about $2.1 \%$ of the symbol length is also added. The bias current is set at 9 $\mathrm{mA}$, while the modulation depth is fixed at $450 \mathrm{mV}$ in order to limit the frequency chirp insurgence and reduce the penalty due to SSB filtering. The DMT signal is generated by a MICRAM 100-GS/s digital-to-analog converter (DAC10002) with 35-GHz electrical bandwidth and 6 bits vertical resolution and it directly modulates the VCSEL with a bias current of $9 \mathrm{~mA}$ and a modulation depth of at $450 \mathrm{mV}$. The modulation depth is optimized in order to limit the spectrum broadening associated with the frequency chirp, reducing the penalty due to SSB and WSSnode filtering. The SSB modulation is obtained by properly detuning a standard 25-GHz spacing WSS filter, which shows a 21-GHz full width half maximum (FWHM) [19]; the frequency detuning, around 8GHz, allows to select half of the DMT dual sideband (DSB) spectrum while preserving the optical carrier, as shown in Figure 2 a).

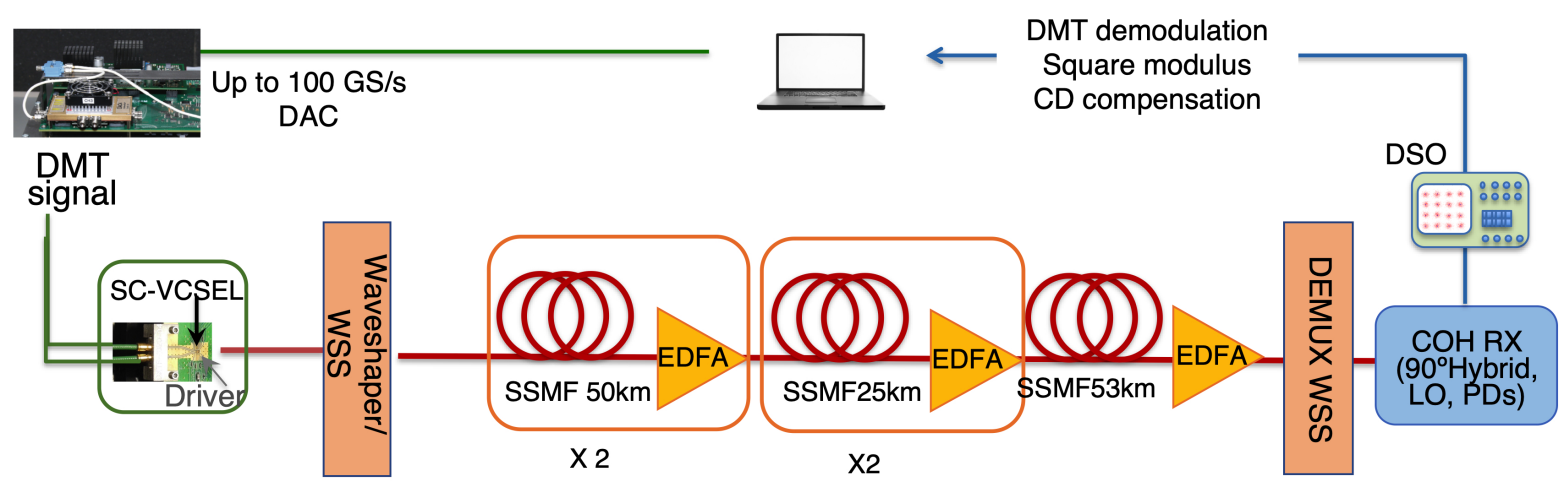

Figure 1. Experimental set up employed to perform a preliminary assessment of the VCSEL-based multi$\mathrm{Tb} / \mathrm{s}$ transmitter.

After amplification in an Erbium-doped fibre amplifier (EDFA), the generated SSB DMT signal is transmitted in the emulated MAN link, constituted by 5 SSMF spools, to bridge up to $200 \mathrm{~km}$ distance. The launch power is set to $+1 \mathrm{dBm}$.

At the receiver side in order to target hundreds of kilometres of transmission distances, we employ digital coherent detection (COHD) which allows easy CD DSP compensation, avoiding also the VCSEL chirp interplay with CD. The chirp associated with VCSEL DM will impair transmission performance only due to the presence of filtering associated to wavelength multiplexers and demultiplexers. Actually, the received signal is detected by a Tektronix coherent receiver OM4245 with $45-\mathrm{GHz}$ bandwidth; the local oscillator (LO) is a tuneable $100-\mathrm{kHz}$ laser with $+15.5 \mathrm{dBm}$ optical power. The inphase/quadrature signals are acquired by a Tektronix real-time oscilloscope with 8-bits vertical resolution, 100-GS/s and 33-GHz electrical bandwidth respectively. Alternatively, some preliminary back to back measurements have been performed with DD obtained with a pre-amplified 50GHz PIN photodiode (PD). After ADC conversion the signal is processed off-line. Due to DM, the transmitted signal is just intensity modulated and then a simplified coherent receiver can be used: after I and Q components recovery and $\mathrm{CD}$ compensation the I and Q square moduli are performed and summed up in order to obtain the originally transmitted intensity signal. This approach has the drawback to cancel the COHD advantages in terms of bit error rate (BER) as a function of signal to noise ratio (SNR) with respect to direct detection (DD), but avoids the use of phase and frequency recovery, reducing the complexity of the receiver DSP and also relaxing the 
constraints on VCSEL and local oscillator (LO) linewidths [14]. The signal to noise ratio (SNR) of each sub-carrier is obtained by transmitting a probe DMT signal, mapped with uniform QPSK loading and it is used to for perform Chow's algorithm, as standard bit- and power-loading procedure [20]; the target bit error rate (BER) is set to $4.6 \cdot 10^{-3}$ according with the exploitation of an advanced hard-decision forward error correction code with $7 \%$ overhead.

\section{EXPERIMETAL RESULTS}
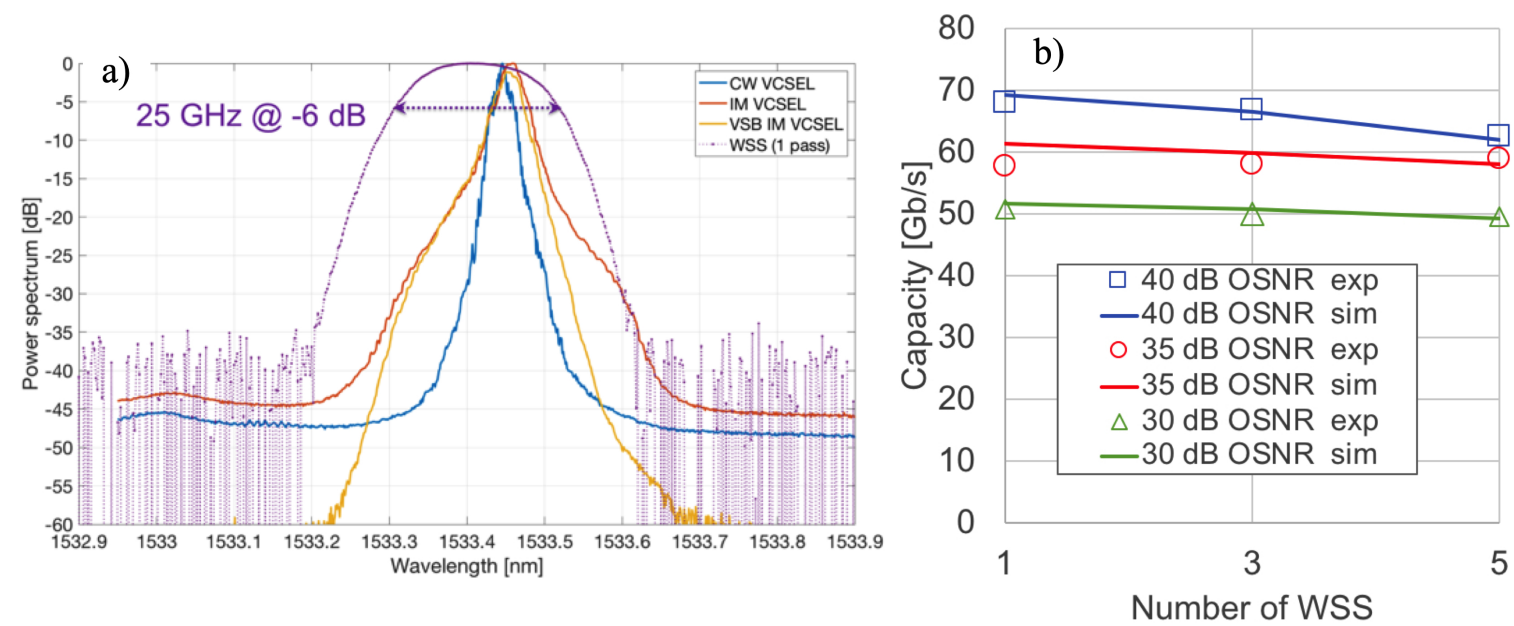

Figure 2 a) SSB DMT spectra: CW blue, DSB DMT orange, SSB DMT yellow, WSS purple. b) transmission capacities in b2b vs number of crossed WSS for: 40-dB OSNR (blue), 35-dB OSNR (red), 30-dB OSNR (green), simulations (continuous line) experimental results (open symbol).

At first we performed some preliminary back to back (b2b) measurements with DD and we compared them with simulations obtained with a transmission model including the electro/optical and chirp transfer functions of the VCSELs, the adopted receiver and featuring all experimental conditions including bias current, modulation depth, electrical device bandwidths, DACs and ADCs bit resolution, PD noise equivalent current (NEC), received powers and the measured WSS transfer function. Fig. 2b) shows the b2b measured capacities achieved for optical SNR (OSNR) ranging from $30 \mathrm{~dB}$ to $40 \mathrm{~dB}$ in function of the number of crossed WSS (open symbols); experimental results obtained by a variable optical noise loader are compared with simulations (continuous line). As expected, a slight reduction of the capacity appears for lower OSNRs, whereas the capacity barely reduces increasing the number of crossed WSS. Thanks to the steep profile of the WSS transfer function, in fact, the carrier and the single sideband optical spectrum are preserved even in case of several WSS crossings. A very good agreement is obtained between experimental results and simulations.

We then evaluated the performance of the VCSEL-based S-BVT after SSMF propagation in single-channel single-polarization conditions. Figure 3 shows the measured SSB-DMT capacities as a function of the bridged SSMF length for a launch power of $+1 \mathrm{dBm}$ in every span; on the right the corresponding OSNRs are also displayed. A capacity around $65 \mathrm{~Gb} / \mathrm{s}$ is supported up to $100-\mathrm{km}$ SSMF propagation thanks to high OSNR levels, while it decreases for $150 \mathrm{~km}$ and $200 \mathrm{~km}$. However, a total transported capacity per polarization higher than the target $50 \mathrm{~Gb} / \mathrm{s}$ can still be achieved even after 200-km propagation [21].

Finally, for 100-km and 200-km SSMF reaches we measured the performance in presence of WSS filter cascading, which can impair the directly-modulated DMT signal transmission, due to the filter narrowing effect [15]. In particular in the experiments the multiple crossing of WSS filters has been emulated by the Finisar Waveshaper filter by featuring the transfer function of the cascaded WSSes. Table I presents the percentage reduction of capacity with respect to the b2b condition (e.g. $67 \mathrm{~Gb} / \mathrm{s}$ ) due to propagation in $100 \mathrm{~km}$ and $200 \mathrm{~km}$ SSMF and WSSes crossing. It can be noticed that the capacity barely reduces increasing the number of crossed WSSs, allowing capacities higher than $50 \mathrm{~Gb} / \mathrm{s}$ even after 200-km SSMF propagation and 6 crossed WSS-like network nodes. 


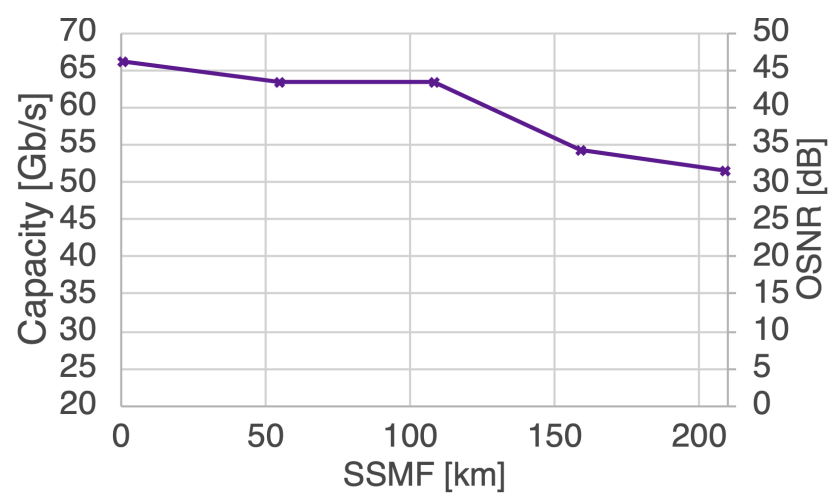

Figure 3 Measured SSB-DMT capacities as a function of the bridged SSMF length [km], on the right the corresponding OSNRs are also reported.

Table I SSB DMT percentage reduction of capacity with respect to the b2b condition due to propagation in 100 $\mathrm{km}$ and $200 \mathrm{~km}$ SSMF as a function of the number of WSSes crossing (up to 6).

\begin{tabular}{|c|c|c|c|}
\hline \multirow{5}{*}{ 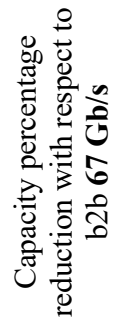 } & Number of WSS & $100 \mathrm{~km} \mathrm{SSMF}$ & $200 \mathrm{~km}$ SSMF \\
\hline & 1 & $6 \%$ & $23 \%$ \\
\hline & 2 & $6 \%$ & $24 \%$ \\
\hline & 4 & $6 \%$ & $25 \%$ \\
\hline & 6 & $7 \%$ & $26 \%$ \\
\hline
\end{tabular}

\section{CONCLUSIONS}

We presented preliminary experimental assessments of DM VCSEL sources with multi-carrier modulation formats, to target hundreds of kilometres transmission in SSMF with per SOP per channel capacity beyond 50 $\mathrm{Gb} / \mathrm{s}$. As multi-Tb/s transmitter modules including multiple C-band DM-VCSELs and WDM multiplexers in SOI chips can be aggregated to target a very fine WDM granularity (i.e. 25-GHz) [16], SSB DMT modulation appears one of the most promising formats to be exploited.

Indeed, the combination with simplified coherent detection allows to bridge MAN typical link distances thanks to DSP CD compensation, but due to the presence of numerous hops between nodes inside metropolitan area networks the effect of filtering has also to be taken into account. For these reasons we analysed the impact on the transmission performance of cascaded WSSes filter, remembering that a spectral broadening is induced by the chirp associated with DM.

The obtained preliminary results confirm that by means of SSB DMT direct modulation and simplified coherent detection 200-km SSMF distances can be reached also in presence of $25-\mathrm{GHz}$ WSS filters with $50-\mathrm{Gb} / \mathrm{s}$ capacity per SOP, enabling 4-b/s/Hz spectral efficiency PDM S-BVT multiple modules for MAN multi-Tb/s transmission.

\section{ACKNOWLEDGEMENTS}

This work has been supported by the H2020 PASSION Project (GA 780326). The authors thank Tektronix, Micram and Opsys Technologies sponsorship.

\section{REFERENCES}

[1] M. S. Moreolo et al., " Spectrum/Space Switching and Multi-Terabit Transmission in Agile Optical Metro Networks," in OECC/PSC 2019 Proceedings, C000235.

[2] J. Renaudier, "100nm ultra-wide optical fiber transmission systems using semiconductor optical amplifiers", Proc. ECOC 2018, paper Mo4G.5 (2018).

[3] J. Lin, et al. "Joint Digital and Optical Pre-compensation for $720 \mathrm{~Gb} / \mathrm{s}$ All-Silicon IQ modulator Single Carrier Transmission", Proc. ECOC 2018, paper We3G.2 (2018).

[4] S. Bhat, et al. "Low Loss Devices fabricated on the Open Access 3mm SOI Waveguide Platform at VTT" in European Conference on Integrated Optics 2019, Ghent, Belgium, 2019, paper T.Po2.18. 
[5] A. Boletti, P. Boffi, P. Martelli, M. Ferrario, M. Martinelli "Performance analysis of communication links based on VCSEL and silicon photonics technology for high-capacity data-intensive scenario" Opt. Express, 23, 1806-1815 (2015).

[6] P. Boffi, A. Gatto, A. Boletti, P. Martelli, M. Martinelli “12.5Gbit/s VCSEL-based transmission over legacy MMFs by centre-launching technique” Electron. Lett., 48, 1289-1290, 2012.

[7] A. Gatto et al., "VCSEL-based communications for metro and access networks," in Proc. OECC/PSC 2018, paper 8751367, September 2018.

[8] A. Gatto et al., "Discrete multitone modulation for short-reach mode division multiplexing transmission," Journal of Lightwave Technology, 37, 20, 5185-5192 (2019).

[9] M. S. Moreolo et al., "Modular SDN-enabled S-BVT Adopting Widely Tunable MEMS VCSEL for Flexible/Elastic Optical Metro Networks," in Proceedings OFC 2018, San Diego, CA, paper M1A.7, March 2018.

[10] C. Xie, et al., "Single VCSEL 100-Gb/s short reach system using discrete multi-tone modulation and direct detection," in Proceedings OFC 2015, Los Angeles, CA, paper Tu2H.2, March 2015.

[11] A. Gatto, et al., "Beyond $25 \mathrm{~Gb} / \mathrm{s}$ Directly-Modulated Widely Tunable VCSEL for Next Generation Access Network," in Proceedings OFC 2018, San Diego, CA, paper Th1E.2, March 2018.

[12] P. Parolari, A. Gatto, C. Neumeyr, and P. Boffi, "Flexible transmitters based on directly modulated VCSELs for next-generation 50G passive optical networks," J. Opt. Commun. Netw. 12, D78-D85 (2020).

[13] C. Xie, S. Spiga, P. Dong, et al., "400-Gb/s PDM-4PAM WDM System Using a Monolithic $2 \times 4$ VCSEL Array and Coherent Detection," J. Lightwave Technol., 33, (3), pp. 670-677, 2015.

[14] P. Parolari, et al., "Effect of filtering in dense WDM metro networks adopting VCSEL-based multi-Tb/s transmitters" 2019 21th International Conference on Transparent Optical Networks (ICTON), Angers, 2019, pp. 1-4.

[15] M. Rapisarda, et al. "Impact of Chirp in High-Capacity Optical Metro Networks Employing DirectlyModulated VCSELs,” Photonics, 5, (4), pp. 51, 2018.

[16] P. Boffi, et al., ""Multi-Tb/s sustainable MAN scenario enabled by VCSEL-based innovative technological solutions", Proc. SPIE 11308, Metro and Data Center Optical Networks and Short-Reach Links III, 113080G (31 January 2020).

[17] M. Svaluto Moreolo, et al. "SDN-enabled sliceable BVT based on multicarrier technology for multi-flow rate/distance and grid adaptation." J. Lightwave Technol., 34, 1516-1522, 2016.

[18] P. Martelli et al., "Polarization Stabilizer for Polarization-Division Multiplexed Optical Systems," 33rd European Conference and Exhibition of Optical Communication, Berlin, Germany, 2007, pp. 1-2.

[19] C. Pulikkaseril, L.A. Stewart, M.A.F. Roelens, G.W. Baxter, S. Poole, S. Frisken, "Spectral modeling of channel band shapes in wavelength selective switches." Opt. Express, 19, 8458-8470, 2011.

[20] P. S. Chow et al., "A practical discrete multitone transceiver loading algorithm for data transmission over spectrally shaped channels," IEEE Trans. on Comm., 43, 773-775, 1995.

[21] A. Gatto, et al. "1.5- $\mu \mathrm{m}$ VCSEL-based system exploiting direct DMT modulation and coherent detection for multi-Tb/s metro link" 2020 International Conference on Optical Network Design and Modeling (ONDM), Barcelona, paper \#1570621755, 2020. 